\title{
Severe Case of Methicillin-resistant Staphylococcus aureus Discospondylitis in a Doberman
}

\author{
MIHAI MUSTEATA ${ }^{1}$, DIANA MOCANU ${ }^{1}$, EUSEBIU SINDILAR ${ }^{2}$, MIHAI MARES ${ }^{3}$, RAMONA FLORINA MORARU ${ }^{3}$, \\ RALUCA NADINA PACURAR ${ }^{5}$, GETA PAVEL ${ }^{4}$, GHEORGHE SOLCAN ${ }^{1}$, ROBERT CRISTIAN PURDOIU ${ }^{5 *}$, RADU LACATUS ${ }^{5}$ \\ ${ }^{1}$ Neurology Clinical Unit, Clinics Department, Veterinary Teaching Hospital, Faculty of Veterinary Medicine, University of \\ Agricultural Science and Veterinary Medicine Ion Ionescu de la Brad lasi, 8 M. Sadoveanu Alley, 700489, Iasi, Romania \\ ${ }^{2}$ Small Animal Surgery, Clinics Department, Veterinary Teaching Hospital, Faculty of Veterinary Medicine, University of Agricultural \\ Science and Veterinary Medicine Ion Ionescu de la Brad lasi, 8 M. Sadoveanu Alley, 700489, Iasi, Romania \\ ${ }^{3}$ Laboratory of Antimicrobial Chemotherapy, Faculty of Veterinary Medicine, University of Agricultural Science and Veterinary \\ Medicine Ion Ionescu de la Brad Iasi, 8 M. Sadoveanu Alley, 700489, Iasi, Romania \\ ${ }^{4}$ Physiology, Basic Science Department, Faculty of Veterinary Medicine, University of Agricultural Science and Veterinary \\ Medicine Ion Ionescu de la Brad Iasi, 8 M. Sadoveanu, Alley, 700489, Iasi, Romania \\ ${ }^{5}$ University of Agricultural Sciences and Veterinary Medicine Cluj Napoca,Laboratory of Radiology, 3-5 Manastur, 400372, Cluj \\ Napoca, Romania
}

\begin{abstract}
A 6-year-old, neutered female Doberman Pinscher was presented with acute progressive paraparesis consistent with T3-L3 myelopathy. For 5 months prior to the admission, the dog had multiple recurrent hyperthermic episodes treated with antibiotics and intermittent corticosteroids for a Borrelia and Ehrlichia co-infection. On survey radiographs and CT of the spine, severe osteoproliferative changes were extensively seen throughout the thoracolumbar vertebral column, and were suggestive of discospondylitis. After cytological and microbiological examinations of the vertebral aspirate a severe Staphylococcus aureus methicillin-resistant discospondylitis was established as a final diagnosis. This is the first case report of discospondylitis due to MRSA in dog without vertebral spine surgery. Treatment with corticosteroids can cover the evolution of discospondylitis until the condition became severe and untreatable.
\end{abstract}

Keywords: dog paraparesis, MRSA, osteomyelitis

Discospondylitis is a common pathology of the spinal cord. The routes of vertebral contamination are represented by direct surgery on the vertebral spine, coexistence of other infectious sites within the body (e.g. dental, urinary bladder, genital apparatus) which imply a hematogenous spread of the infection, or by external contamination (e.g., percutaneous foreign bodies, skin lesions and iatrogenic conditions) [1]. Staphylococcus aureus is a common bacteria implied in discospondylitis [1-3]. Contamination with Staphylococcus aureus methicilin-resistant(MRSA) is well documented in human medicine and rare in veterinary medicine, and occurs mainly as a hospital acquired infection [4-7]. This paper describes an occult and severe case of discospondylitis due to MRSA infection in a dog previously diagnosed and treated for a concurrent co-infection with Borrelia and Ehrlichia.

\section{Experimental part}

\section{Material and methods}

Physical and neurological examination were performed for a 6-year-old neutered female Doberman Pinscher presented to the Veterinary Teaching Hospital (VTH) at lasi Faculty of Veterinary Medicine with acute progressive paraparesis occurred $48 \mathrm{~h}$ prior to presentation. Cell blood count was made with ABC VET analyzer.Serum biochemistry was made with ACCENT Cormay analyzer using specific reagents. A CT scan of the thoracic and lumbar spine was performed (Siemens 16 -slice CT, with a fixed $512 \times 512$ matrix). Contiguous transverse $3 \mathrm{~mm}$ slices were obtained from the level of T3 to L6 vertebrae. For CT examination, the dog was sedated with Medetomidine $(0.03 \mathrm{mg} / \mathrm{kg}$, inj.im) and was positioned in dorsal recumbence. Cytological examination was made on slices after May Grumwald Giemsa staining. Microbiological identification and genetic analysis was made according to previously described methodology (Murakami et al, 1991).

\section{Result and discussions}

A 6-year-old neutered female Doberman Pinscher was presented to the Veterinary Teaching Hospital (VTH) at lasi Faculty of Veterinary Medicine with acute progressive paraparesis occurred $48 \mathrm{~h}$ prior to presentation. The dog suffered a total hysterectomy 5 months before admission, performed in a private clinic. Two weeks after surgery she had a fever episode, when it was diagnosed with Lyme disease and Ehrlichia coinfection and treated with different antibiotics for four months (Clindamycin, Gentamycin, Cefuroxim, Doxycycline, Enrofloxacin) and intermittent corticosteroids ( 3 weeks of Prednisone, $0.5 \mathrm{mg} / \mathrm{kg} / \mathrm{day}$ PO). No X-ray examination was performed. At the time of admission to the VTH, the dog's physical and neurological examination findings included hypertemia $\left(40.8^{\circ} \mathrm{C}\right)$, spinal kyphosis, tense abdominal palpation, preserved deep pain perception and hyperreflexia on the posterior limbs and spinal hyperesthesia. The hematological profile exhibited degenerative, normocytic, normochromic anemia with severe leukocytosis $\left(\mathrm{WBC}=35.5 \times 10^{3} / \mu \mathrm{L}\right)$. No significant changes were present in the biochemical profile of the serum. The neuroanatomic localization of the lesion was consistent with a T3-L3 myelopathy.

On radiographic exam, extensive osteoproliferative changes were extensively seen throughout the thoracolumbar vertebral spine, more obvious caudally to T11, consisting in diffuse idiopathic skeletal hyperostosis 


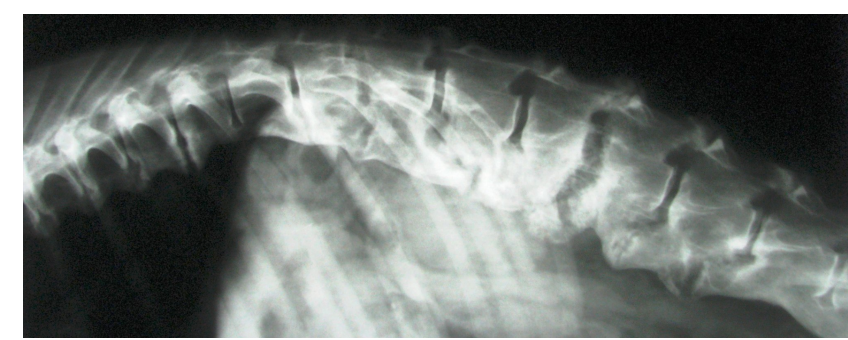

Fig. 1. Thoracolumbar survey radiography, lateral view. Osteoproliferative lesions are present throughout the

thoracolumbar vertebral column, more severe in the lumbar segments

(DISH) (fig. 1) [8]. At thoracic level, a spondyl was found caudally to T6. At T7 to T10 both cranial and caudal aspects of vertebra presented spondyls. From T7 to T10, both cranial and caudal aspects of vertebra presented spondyls. Osteolytic changes were identified at T12-T13 and L2-L3. At the level of T12-T13 intervertebral space, irregularities on vertebral end plates were seen, associated with lytic aspect of spondyls. At T13-L1 intervertebral space, lytic phenomena were detected at the level of ventral bone proliferation, including irregularities and a possible fracture line. Both the caudal end plate of T13 and the cranial end plate of $L 1$ exhibited increased radio-opacity.

On lumbar vertebrae, at the level of L1-L2, a hyperintense area was seen on the ventral bone proliferation. At the level of $L 2-L 3$, the most expressive lesions were present: enlargement of intervertebral space, increased radio-density of caudal $L 2$ vertebral end plate; irregularities on cranial L3 vertebral end plate, irregularnon-homogenous hyper-intensity at the level of intervertebral space. Proliferative lesions were also observed at the level of articular facet for L2 and L3. At L3-L4 level, hyper-intensity of cranio-ventral area of the L 4 and lytic lesions in the vertebral block were present. A diffuse hyper-intense lesion was seen on dorsal articular facets. Irregularities at the level of ventral paravertebral block were present at $L 4$ and L5. Extensive osteo-proliferation was present along the ventral surface of the vertebral spine from T6 through the sacrum, consistent with DISH [8].

Taking into consideration these findings, the diagnosis of severe discospondylitis was suspected. The primary differential diagnoses included infectious osteomyelitis (discospondylitis) and neoplasia.

In order to asses the spinal cord involvement a CT examination was performed. Osteolysis was present with almost complete distortion of the T12-T13 and L2-L3 vertebral bodies. Extradural compression of the spinal cord was presentfrom the caudal third of the $L 3$ vertebral body through the caudal area of L4, those losing the normal vertebral architecture (fig. 2). The compression was thought to be focal invasion by proliferative tissue or purulent material.

A vertebral aspirate and biopsy were performed at the L2-L3 level through invasive surgery. Approximately five milliliters of hemorrhagic purulentfluid was extracted from the site of vertebral body, which was cytologically and microbiologically examined. On cytological exam, nuclear cells (degenerated neutrophils, monocytes, plasmocytes, lymphocytes and macrophages) and cocci agglomerations were observed. No neoplastic characteristics were identified in any of the cells. The isolate was identified as methicillin-resistant Staphylococcus aureus (MRSA) based on its morpho-physiological characteristics and susceptibility pattern. The presumptive identification was confirmed by PCR detection of mecA gene using a method previously described by Murakami et al. [9]

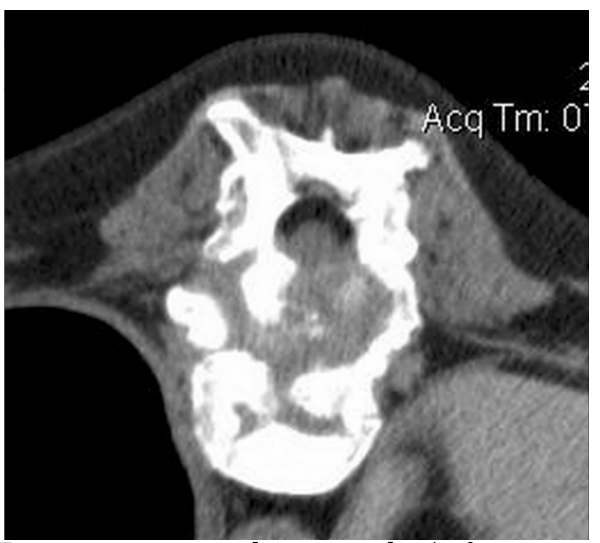

Fig. 2. Transverse computed tomography (soft tissue window) at the level of L2 vertebral body. Normal vertebral architecture is lost because of the marked osteoproliferative and osteolytic lesions. The extradural compression of the spinal cord is caused by the

focal invasion of the infection with proliferative tissue and purulent material

A severe MRSA discospondylitis was established as a final diagnosis. Because of the extension of the lesions, the owner requested euthanasia but declined a necropsy.

This paper describes a severe case of vertebral discospondylitis with methicillin-resistant Staphylococcus aureus (MRSA) in a dog. To this date, there is only one report about discospondylitis due to MRSA secondary to a vertebral spine surgery [10]. To the author's knowledge this is the first report regarding MRSA contamination of the vertebral column in the absence of a direct surgery on the disk or vertebra.

Spinal pain, kyphosis and acute neurologic dysfunction, mainly represented by paraparesis, are common clinical signs in discospondylitis and occur in almost $50 \%$ of the patients [11]. Despite the above-described symptomatology, during the first five months of illness none of the neurological signs appeared in this case. The previously diagnosed diseases (i.e. Borrelia and Ehrlichia infections) of the patient were treated with corticosteroids and various antibiotics. This therapy might have masked the majority of the clinical signs, permitting rapid progression of discospondylitis until it became a severe and untreatable condition [11]. It is well known that the administration of corticosteroids is a trigger factor for the decrease of immunological defense and can accelerate the evolution of discospondylitis [12]. Moreover, because the patient was diagnosed with Lyme disease, clinical sings were difficult to discern. Lyme disease has a polymorphic symptomatology, including both neurological and non-neurological clinical signs [13]. Furthermore, the therapeutic protocols in Lyme disease imply administration of different type of antibiotics for a long period of time [13].

Generally, discospondylitis is diagnosed using survey radiographs, in which lytic and proliferative lesions are seen at the affected sites. Cases as this patient, with regional extension, are more common in neoplastic bone conditions. Vertebral tumors may have the same aspect as osteomyelitis (OM) in radiographic survey [14]. In osteosarcoma (OS), the same clinical signs, except the fever, can occur. Rarely, the OM-OS association was reported [15]. Nevertheless, the presence of a pure $S$. aureus culture and lack of neoplastic-like cells in the vertebral aspirate confirm the diagnosis of discospondylitis, and excludes osteosarcoma.

In this paper we describe the first case report of discospondylitis due to MRSA in dog without vertebral spine surgery. The origin of infection is questionable as far as 
dogs do not naturally carry MRSA. The origin of MRSA discospondylitis in this case should be either contamination from human skin either hospital infection during the spay surgery five months prior diagnosis of discospondylitis. The prolonged antibiotic and steroid therapy likely selected the methicilin resistant strains. In this light we highlight the importance of biosecurity protocols in veterinary practice which have important consequences both in veterinary patients but also for veterinarians.

\section{Conclusions}

To the authors' knowledge, this is the first case report of discospondylitis due to MRSA in dog without vertebral spine surgery. In this case, MRSA infection can be caused by the previous surgical procedure (total hysterectomy) and its hidden evolution was due to Borrelia and Erlichia coinfection and therapy. This therapy might have masked the majority of the clinical signs, permitting rapid progression of discospondylitis until the condition became severe and untreatable.

\section{References}

1.BURKERT BA, KERWIN SC, HOSGOOD GL, PECHMAN RD, FONTENELLE JP: J. Am. Vet. Med. Assoc., 227, nr. 2, 2005, p. 268.

2.DAVIS MJ , DEWEY CW, WALKER MA, KERWIN SC, MOON ML, KORTZ GD, KOBLIK PD, MAHAFFEY MB, BUDSBERG SC, SLATER MR. J . Am. Anim. Hosp. Assoc., 2000, 36 nr. 1, p. 81.

3. KORNEGAY JN, BARBER DL. J. Am. Vet. Med. Assoc., 177, nr. 4, 1980, p. 337.
4. STROMMENGER B, KEHRENBERG C, KETTLITZ C, CUNY C, VERSPOHL J, WITTE W, SCHWARZ S. J. Antimicrob. Chemother., 57, nr. 3, 2006, p. 461.

5.LOEFFLER A, BOAG AK, SUNG J, LINDSAY JA, GUARDABASSI L, DALSGAARD A, SMITH H, STEVENS KB, LLOYD DH. J. Antimicrob. Chemother., 56, nr. 4, 2005, p. 692.

6.BAPTISTE KE, WILLIAMS K, WILLAMS NJ, WATTRET A, CLEGG PD, DAWSON S, CORKILL JE, O'NEILL T, HART CA. Emerg. Infect. Dis., 11, nr. 12, 2005, p. 1942.

7. WEESE J S. J. Am. Anim. Hosp. Assoc., 41, nr. 3, 2005, p. 150. 8.KRANENBURG HC, VOORHOUT G, GRINWIS GC, HAZEWINKEL HA, MEIJ BP. Vet. J., 190, nr. 2, 2011, p. e84.

9.MURAKAMI K, MINAMIDE W, WADA K, NAKAMURA E, TERAOKA H, WATANABE S. J. Clin. Microbiol, 29, nr. 10, 1991, p. 2240.

10.SCHWARTZ M, BOETTCHER IC, KRAMER S, TIPOLD A. J. Small Anim. Pract., 50, nr. 4, 2009, p. 201.

11.BETBEZE C, MCLAUGHLIN R. Vet. Med., 97, nr. 9, 2002, p. 673.

12.LEVINE JM, LEVINE GJ, BOOZER L, SCHATZBERG SJ, PLATT SR, KENT M, KERWIN SC, FOSGATE GT, J. Am. Vet. Med. Assoc., 232, nr. 3, 2008, p. 411.

13. LITTMAN MP, GOLDSTEIN RE, LABATO MA, LAPPIN MR, MOORE GE. J. Vet. Intern. Med., 20, nr. 2, 2006, p. 422.

14.THOMAS WB. Vet. Clin. N. Am. Small., 30, nr. 1, 2000, p. 169. 15.BOSTON S, SINGH A, MURPHY K, NYKAMP S, Vet. Comp. Orthop. Traumatol., 23, nr. 5, 2010, p. 366.

Manuscript received: 6.09 .2018 